\title{
Article
}

\section{Eye movements during visuospatial judgements}

Meadmore, Katie L., Dror, Itiel E., Bucks, Romola S. and Liversedge, Simon Paul

Available at http://clok.uclan.ac.uk/22418/

Meadmore, Katie L., Dror, Itiel E., Bucks, Romola S. and Liversedge, Simon Paul ORCID: 0000-0002-8579-8546 (2011) Eye movements during visuospatial judgements. Journal of Cognitive Psychology, 23 (1). pp. 92-101. ISSN 2044-5911

It is advisable to refer to the publisher's version if you intend to cite from the work. http://dx.doi.org/10.1080/20445911.2011.447256

For more information about UCLan's research in this area go to http://www.uclan.ac.uk/researchgroups/ and search for <name of research Group>.

For information about Research generally at UCLan please go to http://www.uclan.ac.uk/research/

All outputs in CLoK are protected by Intellectual Property Rights law, including Copyright law. Copyright, IPR and Moral Rights for the works on this site are retained by the individual authors and/or other copyright owners. Terms and conditions for use of this material are defined in the policies page.

\section{CLoK}

Central Lancashire online Knowledge www.clok.uclan.ac.uk

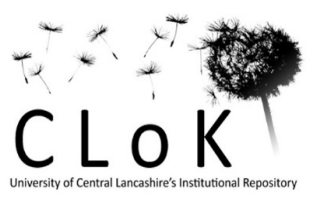




\title{
Eye movements during visuospatial judgements
}

\author{
Katie L. Meadmore ${ }^{1}$, Itiel E. Dror ${ }^{1}$, Romola S. Bucks ${ }^{2}, \&$ Simon P. Liversedge ${ }^{1}$ \\ ${ }^{1}$ School of Psychology \\ University of Southampton \\ Southampton, SO17 1BJ \\ UK \\ ${ }^{2}$ School of Psychology \\ M304, University of Western Australia \\ 35 Stirling Way \\ Crawley 6009, Western Australia
}

Running header: EMs during VS judgements

Send correspondence to: Katie Meadmore

E-mail: klm301@ soton.ac.uk

Tel: 01144 (0) 2380596698

Fax: 01144 (0) 2380594518

School of Psychology

University of Southampton

Southampton, SO17 1BJ

United Kingdom 


\begin{abstract}
The goal of the current research was to determine whether eye movements reflect different underlying cognitive processes associated with visuospatial relation judgements. Ten participants made three different judgements regarding the position of a dot in relation to a bar; an above/below judgement, a near/far judgement, and a precise distance estimation. The results highlight similarities between above/below and near/far visuospatial judgements; specifically, such binary judgements were fast, reflexive and did not require precise distance computation. In contrast, estimating distance was comparatively cognitively demanding and required precise distance computation, as evidenced through distinct scan paths. The eye movement data provide significant insight into the cognitive processes underlying visuospatial judgements, showing aspects of visuospatial processing that are similar, as well as those that differ between tasks.
\end{abstract}


Author Note.

This research was supported by a UK Economic and Social Research Council research studentship to Katie Meadmore. This research was also supported by a UK Economic and Social Research Council research grant (RES 00022 3398) to Simon Liversedge. 
Visuospatial (VS) processing is not unitary and the spatial relations between objects can be computed in numerous ways. For example, describing that the 'cup is on the table' gives a broad categorical description concerning the relative whereabouts of the cup (Kosslyn, 1987; Kosslyn, Chabris, Marsolek, \& Koenig, 1992). However, the table may have a large surface area, and the cup could be located anywhere in this space. The description that the 'cup is near to the corner of the table' provides greater information regarding the location of the cup, but may still not precise enough for successful interaction with the cup. By contrast, specifying ' $5 \mathrm{~cm}$ north east of the bottom left corner of the table' indicates the coordinates of the cup, providing precise distance and directional information (Kosslyn, 1987; Kosslyn et al., 1992).

In the current experiment, we utilised eye movement methodology to investigate the cognitive processes underlying different types of spatial relation judgement. Eye movement recording techniques provide a valuable tool for investigating on-line cognitive processes (Liversedge \& Findlay, 2000; Rayner, 1998). Specifically, eye movement research has significantly increased understanding of the cognitive processes involved in reading, visual search and scene perception (see Castelhano, Mack, \& Henderson, 2009; Rayner, 1998; Rayner \& Castelhano, 2007). For example, increased fixations and longer fixation durations are usually associated with increased difficulty of cognitive processing (Rayner, 1998). Furthermore, it has been clearly shown that patterns of eye movements differ depending on the type of task being undertaken (e.g., Castelhano et al., 2009; Yarbus, 1967). Specifically, there is an extensive literature that shows that differential patterns of eye movements reflect different types of spatial cognition (e.g., Engel, Bertel, \& Barkowsky, 2005; Johansson, Holsanova, Holmqvist, 2006; Kozhevnikov, Motes, \& Hegarty, 2007).

One way spatial relation judgements have been examined is through using a simple bar-dot paradigm, in which participants are presented with a horizontal bar and a dot, which 
is located at varying distances above or below the bar (e.g., Hellige \& Michimata, 1989; Hoyer \& Rybash, 1992; Kosslyn et al., 1989). Typically, participants are asked to make an above/below judgement or a near/far judgement regarding the position of the dot in relation to the bar, and it has been suggested that these two tasks require qualitatively different cognitive processes (see Kosslyn, 1987; Laeng, Chabris, \& Koslyn, 2003). Specifically, binary above/below judgements require discrimination between two possible predetermined patterns; the dot is either above the bar, or is it below the bar, and this judgement is made irrespective of distance. In addition, Kosslyn et al. (1992) argued that the closer the dot is located in relation to the bar, the more difficult it is to make an above/below discrimination judgement.

In contrast, near/far VS judgements require participants to judge whether the dot is within a specific distance of the bar. For example, in Hellige and Michimata's (1989) study, the critical distance was $2 \mathrm{~cm}$, and participants had to judge whether the dot was within or further than $2 \mathrm{~cm}$ from the bar. Dots located at a distance less than $2 \mathrm{~cm}$ were near to the bar, whereas dots located at a distance greater than $2 \mathrm{~cm}$ were far from the bar. Accordingly, near/far tasks are suggested to require some sort of distance judgement.

To be more specific, it is assumed that participants must make a precise distance computation before it is categorised as near or far (Kosslyn et al., 1992; Wilkinson \& Donnelly, 1999). However, this assumption has not been formally examined. Consequently, the extent to which the precise computation of distance is necessary in this situation is unclear (Banich \& Federmeier, 1999; Hoyer \& Rybash, 1992). Indeed, if participants computed the distance from the bar (or dot) by counting out the distance before grouping it into a near/far category, we would expect RT to increase linearly with distance. Alternatively, if participants used the critical distance (i.e. the cut-off boundary for near/far categories) as a reference point and counted away from this towards the dot, we would expect 
the pattern of data to be U-shaped. However, previous research has shown an inverted Ushaped distribution of data (see Sergent, 1991). Thus, it must be considered that near/far judgements may utilise a form of discrimination, much the same as that required for above/below judgements (see Banich \& Federmeier, 1999; Hoyer \& Rybash, 1992; Sergent, 1991; Wilkinson \& Donnelly, 1999). Specifically, the inverted U-shaped distribution may reflect that the discrimination judgement gets more difficult the closer the dot is located to the critical distance.

Consequently, to investigate this hypothesis, in the current study, we recorded eye movements directly to assess the cognitive processes underlying an above/below task, a near/far task, and an additional distance quantification task, in which participants were explicitly required to report the precise distance between a bar and a dot. Specifically, we were interested in whether differential patterns of eye movements reflected qualitatively distinct underlying cognitive processes. We were particularly keen to compare the near/far and distance quantification tasks to determine whether both appear to require precise distance computation. Thus, the present study provides a novel methodological approach to investigate on-line cognitive processing associated with three different types of VS judgements.

In order to make each type of judgement the bar and the dot must be evaluated in relation to each other. Thus, we predicted that, for all three tasks, attention would first be allocated to a reference point; either the bar or the dot. Furthermore, in line with previous research (e.g. Hellige \& Michimata, 1989; Kosslyn et al., 1989), we also anticipated that parafoveal judgements might be made on some occasions, and that these would occur more frequently in the above/below task, less often in the near/far task and very infrequently in the distance quantification task. We made this prediction because we considered distance estimations to be more visuo-cognitively demanding than above/below judgements (and to 
some degree near/far judgements). Thus, we anticipated that participants would be much more likely to directly fixate the stimulus when task demands were high than when they were lower. We also predicted that processing associated with above/below judgements would be qualitatively distinct from processing associated with distance quantification judgements. As such, we anticipated that patterns of eye movements during these two tasks would be different. For the above/below task, we predicted that above/below responses would be made rapidly, would require few fixations on the stimuli and participants would make few errors. We also predicted that the number and duration of fixations would remain relatively constant as the distance of the dot from the bar increased.

For the distance quantification task, given the explicit requirement to form a precise estimate of distance, we anticipated that this would be comparatively cognitively demanding. Accordingly, we expected that participants would make a relatively large number of fixations on the stimuli in this task, and that patterns of eye movements would reflect distance measuring behaviours; such as, counting out units of distance. We also anticipated that estimate accuracy would decrease as a function of distance, and if this was the case, that the number and duration of fixations would increase linearly with distance.

Finally, for the near/far task, we predicted that trials in which the dot was furthest from the critical distance would resemble performance in the above/below task. That is, judgements would be made quickly and with few fixations on the stimuli. By contrast, judgements associated with dots closer to the critical distance were anticipated to be more difficult, with participants taking longer to make responses in these trials. However, a critical question concerns whether the increased RTs might simply reflect more difficult discrimination processes, or instead, processes associated with distance estimation. To discriminate between these possibilities, we predicted that if increased RTs reflected distance estimations, then performance (in terms of eye movements) for judgements when the dot was 
located near to the bar should resemble performance observed for similar distances (i.e. for estimates of 3-6 cm) in the distance quantification task. Alternatively, if increased RTs reflect discrimination processes, then fewer fixations should occur at these distances relative to those that occur in the distance quantification task.

\section{Method}

Participants

Ten right-handed, participants aged between 18-28 years $(M=21.40, S D=3.03)$ volunteered or were awarded course credits for participating in this study. Participants were screened for normal or corrected-to-normal vision. There were 3 males and 7 females.

\section{Design and Materials}

The experiment was programmed using Experiment Builder (SR-Research). Eye movement data were recorded using an EyeLink 1000 eye tracking system. Participants viewed the screen binocularly, but only the movements of the right eye were recorded. Eye movements were recorded during the whole trial; however, analyses were only conducted on eye movement data recorded during the period from the onset of the bar-dot stimulus until the participants made their manual response. To ensure constant viewing conditions, participants used a chin rest and were seated $57 \mathrm{~cm}$ from a 24 inch computer monitor. The stimuli were a $\operatorname{dot}\left(0.6^{\circ} \times 0.6^{\circ}\right)$ and a horizontal bar $\left(5.8^{\circ} \times 0.5^{\circ}\right)$. The stimuli were presented in black on a white screen. A fixation cross $\left(0.5^{\circ} \times 0.5^{\circ}\right)$ was presented in the centre of the screen. Ninetysix trials were presented for each VS task, of which 48 stimuli were presented to the right of the fixation cross, and 48 to the left. Stimuli were lateralised to encourage eye movements from the central position (though note that there was no assessment of visual field 
lateralisation in this study). The edge of the horizontal bar was located at $3.5^{\circ}$ from the fixation cross.

The dot could appear at 1 of 8 distances away from the bar; these were positioned at 1 $\mathrm{cm}\left(1^{\circ}\right)$ increments from the centre of the bar (i.e. $1-8 \mathrm{~cm}$ ); for half of the trials the dot was above the bar, and for half it was below (see Figure 1). The first 4 dots above and below the bar fell within $4.5 \mathrm{~cm}$ of the bar, and the remaining four dots were further than $4.5 \mathrm{~cm}$.

Regions of interest $\left(6.4^{\circ} \times 1^{\circ}\right)$ were set around each distance region in which the dot could be located, above and below the bar. A region of interest was also set around the bar $\left(6.4^{\circ} \times .9^{\circ}\right.$; also see Figure 2 for clarification of the regions of interest). The regions of interest were used to classify the distance from the bar at which participants were fixating.

(Figure 1 about here)

The bar could appear in 1 of 3 locations in on each side of the fixation cross; centrally and slightly above and below central $\left(0.7^{\circ}\right)$. In this way we avoided visual constancy across stimuli (Banich \& Federmeier, 1999). In all three tasks the stimuli were presented in a fixed pseudo-random order, and appeared on one side of the fixation cross in no more than three consecutive trials.

The same stimuli were used for all of the tasks. The above/below task, required an 'above' or 'below' judgement; the near/far task required participants to judge whether the dot was 'within $4.5 \mathrm{~cm}$ ' or 'outside $4.5 \mathrm{~cm}$ ' and the distance quantification task required a distance estimation in $\mathrm{cm}$. 


\section{Procedure}

Before the start of each task, participants' eye movements were calibrated and validated for accuracy. Participants viewed a series of nine dots, presented in three rows at the top, middle and bottom of the screen. Re-calibration occurred throughout the experiment, as necessary.

Each trial within each task consisted of the same sequence of events. A black dot with a white centre appeared in the middle of the screen. Once participants were staring at the white centre of the dot the trial started. The word, 'ready?' appeared centrally. Participants indicated they were ready to begin by pressing a button. A centrally displayed fixation cross appeared for $200 \mathrm{~ms}$, followed by a blank screen (shown for $300 \mathrm{~ms}$ ). The central fixation cross was then displayed again for a further $200 \mathrm{~ms}^{1}$. A bar and dot stimulus then appeared, in either the LVF or RVF. The stimulus remained on the screen until the participant made a response. For the above/below and near/far task, participants pressed one of two buttons. For the distance quantification task, participants pressed a button when they were confident that they had an estimate of the distance between the bar and the dot. This recorded the RT. Participants then typed in their distance estimate using the number pad on the right hand side of a keyboard (the numbers 0-9 and the period, in case they wished to use a decimal). As soon as a response was made, the screen went blank for $300 \mathrm{~ms}$, before a new trial began.

Participants were given verbal instructions before each task began, and a set of eight practice trials, for which they received feedback on incorrect trials. The above/below task was always administered second and the order of the near/far and distance quantification tasks was counterbalanced. 


\section{Results}

Accuracy

For the above/below and near/far tasks there was no ambiguity concerning what constitutes an error. As such, a 2 (Task) x 8 (Distance) ANOVA was conducted on the percentage error rate scores. Consistent with our predictions, there was a main effect of Task, $F(1,9)=13.10, p<.01$. This showed that participants made fewest errors in the above/below task and more errors in the near/far task, suggesting that participants found the above/below task easiest (see Table 1). There was also a main effect of Distance, $F(7,63)=7.17, p<.01$, however, the data in this respect were not particularly clear and comparisons were not significant when Bonferroni corrected. The Task x Distance interaction, $F(7,63)=5.59, p<$ .01 , showed that there was a difference across distance in the near/far task, $F(7,63)=6.72, p$ $<.01$, but not in the above/below task, $F(7,63)=1.48, p<.01$. For the near/far task, errors increased the closer the dot was located to the critical distance $(4.5 \mathrm{~cm})$.

(Table 1 about here)

For the distance quantification task, however, it was less obvious how to categorise a response as erroneous, and so, for this task, accuracy was assessed with respect to the estimates made ${ }^{2}$. Table 1 demonstrates that distance estimate accuracy decreased as the distance of the dot from the bar increased, $F(1,9)=3.99, \mathrm{p}<.01$. However, although numerically the accuracy in estimations decreased with distance, this effect was not reliable when Bonferroni corrected, $t s<3.83$. Overall, it seems reasonable to conclude that participants were quite accurate in estimating distance. 
For RT, number of fixations and average fixation duration, performance was analysed using 3 (Task) x 8 (Distance) repeated measures ANOVAs, followed by planned paired comparisons. Unplanned comparisons were Bonferroni corrected.

\section{Response Time}

In the RT and eye movement analyses for the distance quantification task we analysed the data for all the trials. For the above/below and near/far tasks, analysis was conducted both with data from all the trials, and with only correct responses. We report in full the analysis with only correct responses; however, the pattern of effects was the same when erroneous trials were also included $(F \mathrm{~s}>3.97, p \mathrm{~s}>.01)$.

There were main effects of Task, $F(2,18)=11.68, p<.01$, and Distance, $F(7,63)=$ $5.48, p<.01$. Consistent with our predictions, participants took longest to make a response in the distance quantification task, less time in the near/far task, and least time in the above/below task (see Table 1). Comparisons were not significant across distance when Bonferroni corrected. These main effects were qualified by a Task by Distance interaction, $F(14,126)=3.97, p<.01$. As predicted, the time taken to make an above/below response was short and comparable across distance, $F(7,63)=1.25, n s$. Distance did affect performance in both the near/far task and the distance quantification task $(F \mathrm{~s}>4.12, p \mathrm{~s}<$ .01). Participants were faster to respond in the near/far task when the dot was located at distances 1 or 8 compared to distances 4 or $5(p s<.05)$. The data take the form of an inverted U-shaped curve, indicating that judgements were more difficult the closer the dot was to the critical distance. In contrast, for the distance quantification task, RT increased with distance (judgements at distances 1 and 2 were faster than judgements at distance $8, p$ s < $.05)$. 


\section{Number of fixations}

Unsurprisingly, number of fixations also showed main effects of Task, Distance and a Task x Distance interaction, all $F \mathrm{~s}>11.40, p \mathrm{~s}<.01$. As demonstrated in Table 1, the pattern of results was very similar to that found with the RT data.

\section{Average Fixation Duration}

The main effect of Task, $F(2,18)=23.46, p<.01$, was driven by the longer fixation durations found in the distance quantification task compared to the above/below task and near/far task, $t \mathrm{~s}>4.73, p \mathrm{~s}<.01$. No differences in fixation duration were found between the near/far and above/below tasks, $t(9)=-1.70, n s$. There was also a main effect of Distance, $F(7,63)=3.14, p<.01$, and comparisons showed differences between distances 4 and 8,5 and 8 , and 5 and $7, t \mathrm{~s}>4.61, p s<.001$. These main effects were not qualified by a Task $\mathrm{x}$ Distance interaction, $F(14,126)=.99, n s$.

Average fixation duration can in many situations reflect cognitive processing difficulty (Liversedge \& Findlay, 2000; Rayner, 1998), and so these results indicate that processing for the above/below and near/far task was equivalent and reliably less difficult than processing during the distance quantification task. This effect does not appear to be consistently modulated by distance.

\section{First Fixation Location}

As illustrated in Table 2, 69.2\% of first fixations landed on the bar, distance 1 or distance 2 (computed by summing the mean percentage of fixations for the bar, distance 1 and distance 2 in all three tasks and then dividing by three, the number of tasks). In contrast, 
participants first looked directly at the region in which the dot was located on only $7.0 \%$ of trials (computed by summing the mean percentage of fixations for the dot in all three tasks and then dividing by three). This suggests that participants were most likely to use the bar as a reference point from which a judgement about the dot was then made.

(Table 2 about here)

\section{Scan Patterns}

We categorised scan patterns into seven different types that best represented the patterns of behaviour observed during the tasks: No saccade to stimulus patterns referred to trials in which the participants did not make a saccade to the stimulus and instead remained fixated at the centre of the screen. Fixations made in a single region referred to eye movements from the central cross to land and remain fixated in the same distance region (see Figure 2A). For example, in these trials one or multiple fixations would be made but critically all fixations stayed within the same distance region. Fixations made in two regions with a saccade in between referred to eye movement patterns in which participants made only one saccade to another distance region after the initial fixation on the stimulus (see Figure 2B). Again, in these trials one or multiple fixations could be made in the two distance regions, but critically only one saccade was made from one distance region to the other. The first fixation usually fell within the closest regions to the bar, and then the following saccade was made in the direction of the dot (i.e. away from the bar). Switches were defined as successive eye movements in which the direction of the saccade alternated between being towards the bar and towards the dot (like a zig-zag pattern; see Figure 2C). Multiple fixations in the same direction referred to a series of two or more saccades in the same direction that were one or two distance regions apart (see Figure 2D). In some trials this pattern was followed by a long 
saccade back to the start of the scan path (usually towards the bar), after which multiple fixations in the same direction were started again. Combinations of switches and multiple fixations in the same direction were defined as more complex patterns of eye movements that included scan patterns of both types (see Figure 2E). Finally, other referred to any remaining uncategorised trials (see Figure 2F).

In all these trials, if multiple fixations were made within the same distance region, for the purpose of classifying patterns, they were included as 'one' fixation. Critically, the patterns of saccades depict saccades made from one interest region to another. Since the differences in patterns of scanning were most discriminable when the distance between the bar and the dot was greater, we only considered trials in which the bar and the dot were 5-8 cm apart.

(Figure 2 about here)

Two things are clear from the saccadic patterns. First, there was considerable individual variability across participants; for example, participant 9 favoured 'multiple fixations in the same direction' scan patterns, whereas participant 6 made predominantly 'switches' (see Figure 3). Despite this, performance in terms of accuracy was similar for all participants, suggesting that no one strategy was more effective than another. Second, patterns were qualitatively different in the distance quantification task compared to those in the above/below and near/far tasks (see Figure 3). That is, the most striking finding from these analyses was that for the distance quantification task there was a much higher prevalence of switches, multiple fixations in the same direction and combinations of the two than was the case for the above/below and near/far task $(z \mathrm{~s}>2.52)$. For the above/below and 
near/far tasks no saccade and fixations in one or two regions were more prevalent $(z \mathrm{~s}>2.71)$, especially in the above/below task.

These data indicate that above/below and near/far judgements do not necessarily entail the computation of precise distance, and can (at least on some trials) be made parafoveally. In the distance quantification task, participants always made a saccade from the centre of the screen to the stimulus, and demonstrated distinct distance measuring behaviours.

(Figure 3 about here)

\section{Discussion}

The aim of this experiment was to examine the cognitive processes underlying three different VS judgement tasks, through recording eye movements. There were two key findings. First, we found a quantitative difference in performance measures in the task that explicitly required distance estimation compared to a task in which previous research assumed required precise distance computation. The second key finding was that different scan patterns were found during the distance quantification task compared to both the above/below and near/far judgement tasks. Since eye movements often reflect cognitive processing (Liversedge \& Findlay, 2000), this suggests that qualitatively different cognitive processes may underlie distance estimation compared to above/below and near/far VS judgements.

Above/below and near/far judgements were rapid and often appeared reflexive whereas distance estimation was more cognitively demanding, as demonstrated by an increased number of fixations, longer fixation durations and different patterns of oculomotor behaviour. Furthermore, precise distance computation was essential in the distance quantification task (i.e., participants could not estimate distance from a brief visual 
inspection), and the data were at least consistent with the suggestion that participants often performed distinct scan patterns when computing distance (such as, multiple fixations in the same direction and switches).

As hypothesised, for the near/far task, when the dot was furthest away from the critical distance (i.e. distances 1, 2, 7 and 8) processing resembled that found for above/below VS judgements. That is, judgements were made quickly and with few eye movements. In contrast, and as predicted, RTs were longer when the dots were nearest to the critical distance indicating that these trials were more cognitively demanding. Despite this, however, there was little evidence to suggest that participants undertook similar processing to that observed in the distance quantification task. Not only were the RTs, along with number and durations of fixations, greatly reduced in the near/far task compared to the distance quantification task, but also very different patterns of scanning occurred. Specifically, for example, very few patterns of eye movements consistent with distance measuring behaviours (such as switches and multiple fixations in the same direction) were observed for this task.

Note that some of the effects that we report could be due to increased response choice in the distance quantification task rather than increased task demand. However, we believe that our results are very largely driven by increased task demand based on the eye movement results. Specifically, our finding that participants make more and longer fixations in the distance quantification task than in the above/below or near/far task can be interpreted as evidence of increased cognitive demand (Liversedge \& Findlay, 2000; Rayner, 1998).

It is also important to note that it is unlikely that the differences in task demand between the distance quantification task and the near/far task explain all the differences in the eye movement patterns. Indeed, task demands also differed between the near/far and above/below tasks. For example, eye movements differed with respect to distance, and participants made more fixations and took longer to respond in the near/far compared to the 
above/below task. However, it would seem that these differences were quantitative and not qualitative, as patterns of scanning were quite similar.

Critically, we argue that if near/far spatial relation judgements required precise distance computation before classification into near/far categories, similar eye movement behaviour to that observed in the distance quantification task should have occurred. Thus, we have shown both quantitative and qualitative differences between processing in distance quantification and near/far judgement tasks, indicating differences in both task demand and the underlying cognitive processes. In addition, it seems reasonable to conclude that precise distance was not necessarily computed for near/far or above/below VS judgements and such judgements are categorical in nature. This is in contrast to previous studies in which above/below and near/far judgements are suggested to be independent VS processes (for a review, see Laeng et al., 2003).

In conclusion, the current experiment has provided significant insight into the cognitive processes underlying spatial relation judgements through the use of eye movement methodology. The results demonstrate both quantitative and qualitative differences in cognitive processing for different VS tasks. Specifically, the results suggest that cognitive processing associated with above/below and near/far judgements is often qualitatively different from that which occurs when the task requires precise distance estimation. 


\section{References}

Banich, M. T., \& Federmeier, K. D. (1999). Categorical and metric spatial processes distinguished by task demands and practice. Journal of Cognitive Neuroscience, 11, 153-166.

Castelhano, M. S., Mack, M. L., \& Henderson, J. M. (2009). Viewing task influences eye movement control during active scene perception. Journal of Vision, 9, 1-15.

Engel, D., Bertel S. \& Barkowsky T. (2005). Spatial Principles in Control of Focus in Reasoning with Mental Representations, Images, and Diagrams. Spatial Cognition IV, 181-203.

Hellige, J. B., \& Michimata, C. (1989). Categorization versus distance: Hemispheric differences for processing spatial information. Memory and Cognition, 17, 770-776.

Hoyer, W. J., \& Rybash, J. M. (1992). Age and visual field differences in computing visualspatial relations. Psychology and Aging, 7, 339-342.

Johansson, R., Holsanova, J. \& Holmqvist, K. (2006). Pictures and spoken descriptions elicit similar eye movements during mental imagery, both in light and in complete darkness. Cognitive Science, 30 1053-1079.

Kozhevnikov, M., Motes, M., Hegarty, M. (2007) Spatial visualization in physics problem solving. Cognitive Science, 31, 549-579.

Kosslyn, S. M. (1987). Seeing and imaging in the cerebral hemispheres: A computational approach. Psychological Review, 94, 148-175.

Kosslyn, S. M., Chabris, C. F., Marsolek, C. J., \& Koenig, O. (1992). Categorical versus coordinate spatial relations: Computational analyses and computer simulations. Journal of Experimental Psychology: Human Perception and Performance, 18, 562577. 
Kosslyn, S. M., Koenig, O., Barrett, A., Cave, C., Tang, J., \& Gabrieli, J. D. E. (1989). Evidence for two types of spatial representations: Hemispheric specialization for categorical and coordinate relations. Journal of Experimental Psychology: Human Perception and Performance, 15, 723-735.

Laeng, B., Chabris, C. F., \& Kosslyn, S. M. (2003). Asymmetries in encoding spatial relations. In K. Hugdahl, \& R. J. Davidson (Eds.), The Asymmetrical Brain (pp.303-340). Cambridge, MA: The MIT Press.

Liversedge, S. P., \& Findlay, J. M. (2000). Saccadic eye movements and cognition. Trends in Cognitive Sciences, 4, 6-14.

Rayner, K. (1998). Eye movements in reading and information processing: 20 years of research. Psychological Bulletin, 124, 372-422.

Rayner, K., \& Castelhano, M. S. (2007). Eye movements during reading, scene perception, visual search, and while looking at print advertisements. In R. Pieters, \& M. Wedel (Eds.), Visual advertising. Hillsdale, NJ: Erlbaum.

Sergent, J. (1991). Judgments of relative position and distance on representations of spatial relations. Journal of Experimental Psychology: Human Perception and Performance, $91,762-780$.

Wilkinson, D., \& Donnelly, N. (1997). The role of stimulus factors in making categorical and coordinate spatial judgments. Brain and Cognition, 39, 171-185.

Yarbus, A. L. (1967). Eye movements and vision. New York, Plenum Press. 
Footnotes.

${ }^{1}$ This portion of the procedure was adopted to ensure methodological consistency with other experiments conducted as part of a $\mathrm{PhD}$ thesis.

${ }^{2}$ It must be noted that participants used decimals to estimate distance on $13 \%$ of trials. Thus, although participants were permitted to type in distance estimates as decimals, on the majority of trials they did not. 


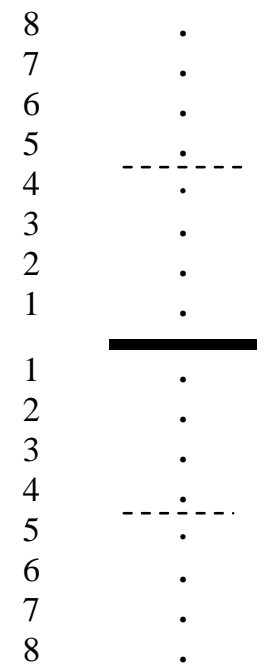

Figure 1. Positions (1-8) in which the dots (•) could appear in relation to the bar (_); (- - ) denotes the boundary for near/far judgements (distances between the bar and the boundary $=$ near; distances further from this boundary $=$ far). 


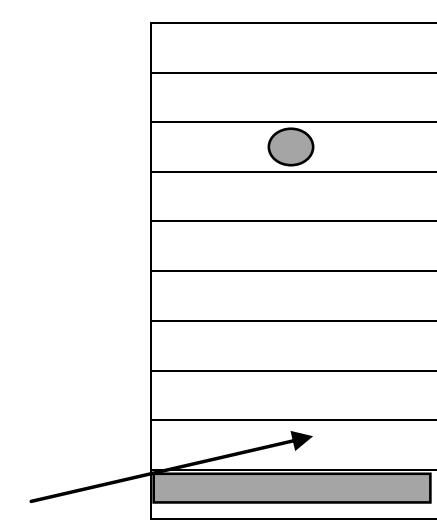

A. Fixation in one distance region

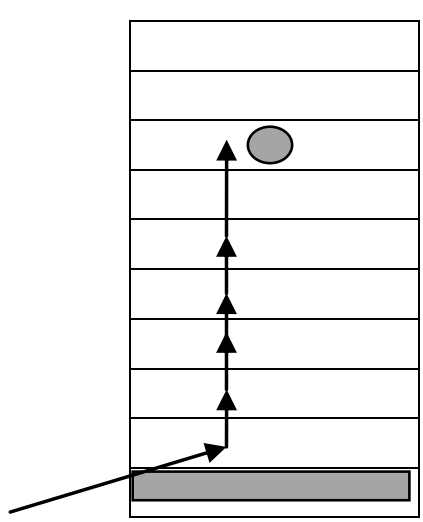

D. Multiple fixations in the same direction

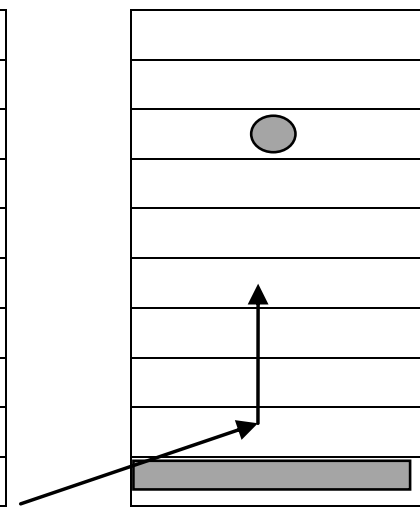

B. Fixation in two distance regions

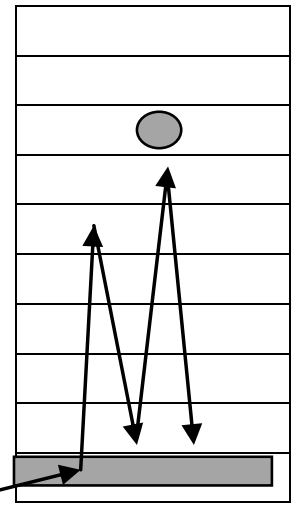

C. Switch

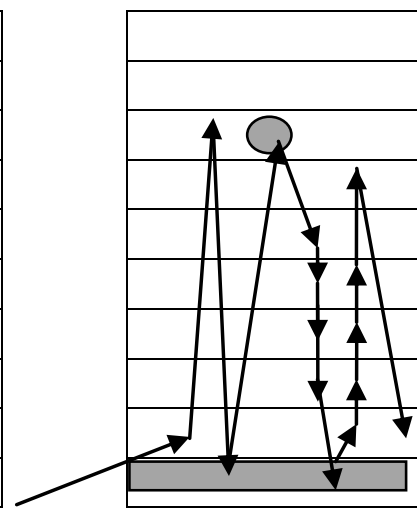

E. Combination of

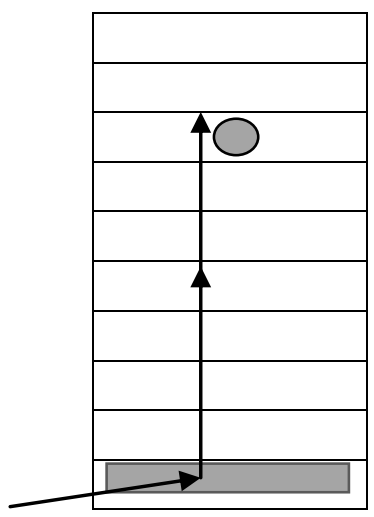

F. Other switch and count

Figure 2. Pictorial example of scan patterns. Note that bars and dots were presented in black and the distance regions could not be seen by the participants. 


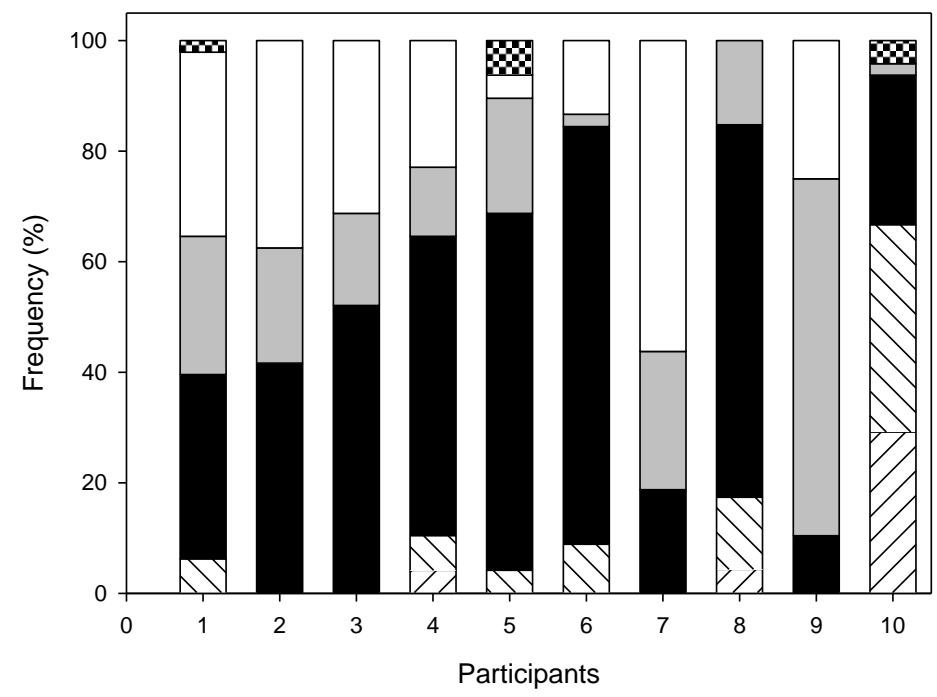

No saccade

$\square$ Fixation in one region

$\triangle \backslash$ Fixation in two regions

Switch

Multiple fixations in the same direction Combination

W00 Other

A. Distance Quantification Task

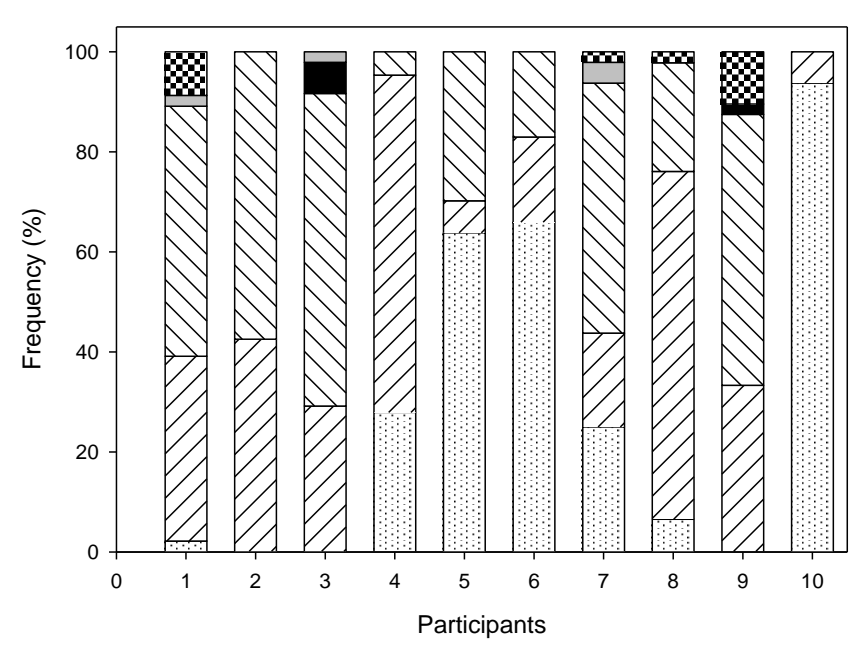

B. Above/Below Task

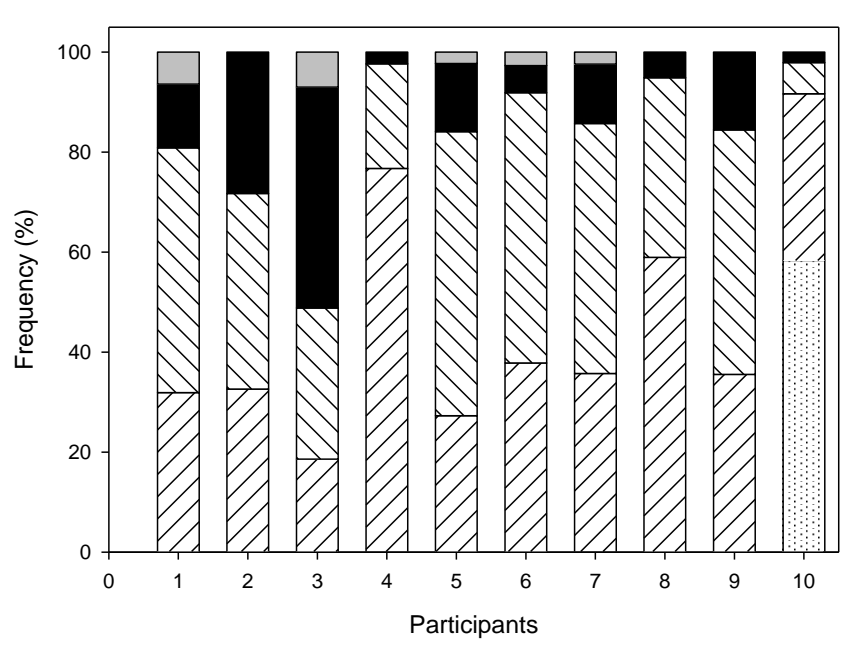

C. Near/Far Task

Figure 3. Scan patterns categorised into seven different types for trials in which the dot appeared 5-8 cm from the bar for each participant in each task. 
Table 1.

Performance Measures as a Function of Task and Distance.

\begin{tabular}{|c|c|c|c|c|c|c|c|c|c|c|c|c|c|c|c|c|c|c|}
\hline & \multicolumn{2}{|c|}{ Distance 1} & \multicolumn{2}{|c|}{ Distance 2} & \multicolumn{2}{|c|}{ Distance 3} & \multicolumn{2}{|c|}{ Distance 4} & \multicolumn{2}{|c|}{ Distance 5} & \multicolumn{2}{|c|}{ Distance 6} & \multicolumn{2}{|c|}{ Distance 7} & \multicolumn{2}{|c|}{ Distance 8} & \multicolumn{2}{|c|}{ Overall } \\
\hline & $M$ & $S D$ & $M$ & $S D$ & $M$ & $S D$ & $M$ & $S D$ & $M$ & $S D$ & $M$ & $S D$ & $M$ & $S D$ & $M$ & $S D$ & $M$ & $S D$ \\
\hline & \multicolumn{18}{|c|}{$\underline{\operatorname{ER}(\%)}$} \\
\hline Above/below & 0 & 0 & 3 & 4.0 & 0 & 0 & 1 & 2.6 & 3 & 5.6 & 4 & 5.9 & 1 & 2.6 & 3 & 5.6 & 2 & 1.6 \\
\hline \multirow[t]{2}{*}{ Near/far } & 1 & 2.6 & 1 & 2.6 & 2 & 3.5 & 13 & 16.8 & 34 & 34.6 & 6 & 10.4 & 1 & 2.6 & 2 & 3.5 & 7 & 5.4 \\
\hline & \multicolumn{18}{|c|}{ Estimate accuracy (absolute difference) } \\
\hline \multirow[t]{2}{*}{ Distance } & 0.1 & 0.1 & 0.3 & 0.3 & 0.3 & 0.3 & 0.3 & 0.2 & 0.3 & 0.3 & 0.5 & 0.3 & 0.6 & 0.4 & 0.6 & 0.4 & .4 & .06 \\
\hline & \multicolumn{18}{|c|}{$\underline{\mathrm{RT}}(\mathrm{ms})$} \\
\hline Above/below & 540.4 & 122.0 & 537.4 & 105.7 & 513.4 & 84.7 & 516.5 & 115.1 & 543.5 & 127.4 & 526.3 & 103.2 & 536.8 & 105.2 & 552.0 & 139.5 & 533.3 & 108.1 \\
\hline Near/far & 672.6 & 139.5 & 715.7 & 207.2 & 877.8 & 315.8 & 1309.2 & 786.6 & 1224.3 & 432.2 & 929.2 & 355.1 & 810.0 & 251.9 & 709.5 & 138.3 & 906.0 & 283.9 \\
\hline \multirow[t]{2}{*}{ Distance } & 2363.3 & 1770.4 & 2558.3 & 1822.9 & 3247.6 & 2211.9 & 3316.0 & 2607.8 & 3225.3 & 2594.1 & 3838.7 & 2819.0 & 3515.6 & 2859.0 & 3932.8 & 3236.1 & 3249.7 & 2442.9 \\
\hline & \multicolumn{18}{|c|}{$\underline{\text { Number of Fixations }}$} \\
\hline Above/below & 2.2 & .6 & 2.2 & .7 & 2.2 & 6 & 2.3 & .8 & 2.3 & .8 & 2.3 & .8 & 2.4 & .8 & 2.4 & .6 & 2.3 & .7 \\
\hline Near/far & 2.8 & .5 & 2.7 & .6 & 3.0 & .8 & 3.7 & 1.5 & 3.7 & 1.1 & 3.4 & 1.1 & 3.2 & .9 & 3.1 & .8 & 3.2 & .8 \\
\hline Distance & 4.6 & 1.7 & 5.0 & 1.9 & 6.4 & 2.4 & 7.0 & 3.3 & 7.2 & 3.3 & 8.4 & 4.0 & 8.8 & 4.4 & 9.2 & 4.9 & 7.1 & 3.1 \\
\hline \multicolumn{19}{|c|}{$\underline{\text { Average Fixation Duration (ms) }}$} \\
\hline Above/below & 221.7 & 44.5 & 226.0 & 61.60 & 229.6 & 58.6 & 229.1 & 58.1 & 229.5 & 73.5 & 228.5 & 73.1 & 218.2 & 64.6 & 217.1 & 67.3 & 224.9 & 60.6 \\
\hline Near/far & 236.3 & 29.2 & 246.8 & 54.38 & 256.5 & 44.0 & 266.2 & 50.6 & 264.1 & 62.2 & 247.1 & 64.9 & 239.1 & 60.8 & 234.2 & 63.3 & 248.8 & 49.7 \\
\hline Distance & 348.4 & 71.1 & 372.6 & 70.0 & 392.0 & 65.4 & 382.6 & 69.0 & 374.1 & 63.2 & 380.0 & 60.9 & 367.0 & 73.9 & 351.6 & 67.0 & 370.2 & 62.7 \\
\hline
\end{tabular}


Table 2

Percentage of first fixations made in each task

Landing Task

position Above/Below Near/Far Distance

\begin{tabular}{lcccccc}
\hline & $M(\%)$ & $S D$ & $M(\%)$ & $S D$ & $M(\%)$ & $S D$ \\
\cline { 2 - 7 } Bar & 16.3 & 14.5 & 13.7 & 6.8 & 17.6 & 11.8 \\
Distance 1 & 28.1 & 10.6 & 37.4 & 12.4 & 32.8 & 8.2 \\
Distance 2 & 19.3 & 5.7 & 20.8 & 4.9 & 21.7 & 4.7 \\
Distance 3 & 10.8 & 6.2 & 11.1 & 5.6 & 12.0 & 6.3 \\
Distance 4 & 10.6 & 5.3 & 8.7 & 5.8 & 7.6 & 3.9 \\
Distance 5 & 6.1 & 4.9 & 4.1 & 2.4 & 4.97 & 2.6 \\
Distance 6 & 4.3 & 3.0 & 3.3 & 2.8 & 2.1 & 2.9 \\
Distance 7 & 3.5 & 3.8 & 0.7 & 1.1 & 1.2 & 1.4 \\
Distance 8 & 1.0 & 1.3 & 0.2 & 0.5 & 0.1 & 0.3 \\
Dot & 7.8 & 7.1 & 6.5 & 7.2 & 6.8 & 4.2 \\
\cline { 2 - 7 } & & & & & & \\
\hline
\end{tabular}

Note. data refer to trials in which a saccade was made. Distance $=$ Distance Quantification task. Data for Distances 1-8 correspond to trials in which the dot was positioned at a different distance. Bar corresponds to the proportion of initial fixations made directly on the Bar, and Dot corresponds to the proportion made directly on the dot regardless of which distance it appeared at. The boxes indicate fixations from which the first fixation location data were computed. 
